Proc Indian Natn Sci Acad $\mathbf{8 1}$ No. 1 February 2015 Special Issue, pp. 174-178

(C) Printed in India.

DOI: $10.16943 /$ ptinsa/2015/v81i1/48066

\title{
Modified Surface Tension of a QGP-Droplet Under One Loop Correction in Peshier Potential
}

\author{
S SOMORENDRO SINGH ${ }^{1}$, AGAM K JHA ${ }^{* 2}$ and K K GUPTA ${ }^{3}$ \\ ${ }^{1}$ Department of Physics and Astrophysics, University of Delhi, Delhi 110 007, India \\ ${ }^{2}$ Department of Physics, Bose Institute, Kolkata 700 009, India \\ ${ }^{3}$ Department of Physics, Kirori Mal College, University of Delhi, Delhi 110 007, India
}

(Received on 1 May 2014; Accepted on 23 June 2014)

\begin{abstract}
Under one loop correction in Peshier potential surface tension of a Quark-Gluon Plasma (QGP) droplet has been recomputed. The correction reduces the stable size of a QGP droplet. The value of surface tension obtained here is in better agreement with the current lattice result.
\end{abstract}

Key Words : Quark-Gluon Plasma; Quark-Hadron Phase Transition

\section{Introduction}

It is well known that under extreme conditions of hadronic density and/or temperature the hadronic system would split into its fundamental constituents, quarks and gluons, such that the bulk properties of the hadronic system would be governed by these degrees of freedom (Shuryak, 1973). Such a (locally) thermally equilibrated state of matter in which quarks and gluons are deconfined from hadrons, so that color degrees of freedom become manifest over inter nuclear distances rather than just intra nucleonic distances, is called Quark-Gluon Plasma (QGP) (Satz, 1978). The phase transition (Csernai et al., 2003; Mustafa et al., 1998; Ramanathan et al., 2007; Shukla and Mohanty, 2001) might be anticipated during Ultra Relativistic Heavy Ions Collisions (URHIC). So ongoing experiments related to URHIC at RHIC and LHC are much more concerned for the new state.

Under a phase transition, the critical free energy difference between the two phases with the help of liquid drop model is given (Kapusta et al., 1995) as:

$$
\begin{gathered}
\Delta F=-\frac{4 \pi}{3} R^{3}\left[P_{h a d}(T)-P_{q, g}(T)\right] \\
+4 \pi R^{2} \sigma+\tau_{c r i t} T \ln \left[1+\left(\frac{4 \pi}{3}\right) R^{3} s_{q, g}\right] .
\end{gathered}
$$

*Author for Correspondence : E-mail: agamjha_2001@yahoo.co.in 
The surface tension is calculated by minimizing the expression (1) with respect to the droplet size. It is obtained as (Linde, 1983):

$$
R_{c}=\frac{2 \sigma}{\Delta p} \text { or } \sigma=\frac{3 \pi \Delta F}{4 \pi R_{c}^{2}} .
$$

In this paper, we briefly revisit free energy incorporating one loop correction factor in the mean-field potential and compute, once again, the surface tension of stable droplets. The perturbative part modifies the density of state of the constituent particles of QGP.

\section{Potential with one Loop Correction and DOS}

The density of states (DOS) of QGP droplet is determined adapting a phenomenological confining potential $V_{\text {conf }}(k)$. The potential is evaluated by considering the interactions between the constituent particles. The calculation is considered with first order correction factor in the potential called mean field potential in phase space. The correction is done in such a way that the expansion of strong coupling constant is perturbed with a loop correction between quark-antiquark and quark-gluons (Ramanathan et al., 2007; Shukla and Mohanty, 2001; Brambilla et al., 2001). It is calculated through the Hamiltonian of system. So the interacting mean field potential is expressed as:

$$
V_{\text {conf }}(k)=(2 \pi / k) \beta \alpha_{s}(k) T^{2}\left[1+\frac{\alpha_{s}(k)}{4 \pi} a_{1}\right]-\frac{m_{0}^{2}}{2 k},
$$

where

$$
\beta=\sqrt{2} \times \sqrt{\left(1 / \beta_{g}\right)^{2}+\left(1 / \beta_{q}\right)^{2}}
$$

called the effective rms value of parametrization factor of $\beta_{q}=1 / 8$ and $\beta_{g}=(8-10) \beta_{q}$. These factors determine the dynamics of QGP flow and subsequent transformation to the confining colorless hadrons. $\alpha_{s}(k)$ is the coupling value of quark and gluon with degree of freedom $n_{f}$,

$$
\alpha_{s}(k)=\frac{4 \pi}{\left(33-2 n_{f}\right) \ln \left(1+k^{2} / \Lambda^{2}\right)}
$$

in which QCD parameter is defined as $\Lambda=0.15 \mathrm{GeV}$. The coefficient $a_{1}$ is one loop correction in the interactions (Fischler, 1977; Billoire, 1980) and it is given as:

$$
a_{1}=2.5833-0.2778 n_{l}
$$

where $n_{l}$ is considered with the number of light quark elements (Smirnov et al., 2008).

So the density of states in phase space with one loop correction factor in the potential is obtained through Ramanathan et al., (Ramanathan et al., 2007; Shukla and Mohanty, 2001; Ramanathan et al., 2004) as:

$$
\int \rho_{q, g}(k) d k=\frac{\nu}{\pi^{2}}\left[-V_{\text {conf }}(k)\right]^{2} \frac{d V_{\text {conf }}}{d k}
$$


or,

$$
\rho_{q, g}(k)=\frac{\nu}{\pi^{2}}\left[\frac{\beta_{q, g}^{3} T^{2}}{2}\right]^{3} g^{6}(k) A
$$

where,

$$
A=\left\{1+\frac{\alpha_{s} a_{1}}{\pi}\right\}^{2}\left[\frac{\left(1+\alpha_{s} a_{1} / \pi\right)}{k^{4}}+\frac{2\left(1+2 \alpha_{s} a_{1} / \pi\right)}{k^{2}\left(k^{2}+\Lambda^{2}\right) \ln \left(1+\frac{k^{2}}{\Lambda^{2}}\right)}\right],
$$

$\nu$ is the volume occupied by the QGP and $k$ is the relativistic four-momentum in natural units and $g^{2}(k)=$ $4 \pi \alpha_{s}(k)$.

\section{The Free Energy Evolution and Surface Tension}

With the help of free energies (Peshier et al., 1994; Smirnov et al., 2008; Ramanathan et al., 2004; Neegaard and Madsen, 1999; Christiansen and Madsen, 1997; Balian and Block, 1970; Marder and Sretitsky, 1991; Singh and Ramanathan, 2013; Iwasaki et al., 1994) under the loop correction, the surface tension is computed as

Table 1: Surface tension of QGP droplet at $\beta_{g}=8 \beta_{q}, \beta_{q}=1 / 8$;

\begin{tabular}{|r|r|r|r|c|}
\hline$T_{c}$ & $\begin{array}{r}\Delta F_{c} \\
(\mathrm{MeV})\end{array}$ & $\begin{array}{r}R_{c} \\
(\mathrm{MeV})\end{array}$ & $\begin{array}{r}\sigma \\
(\mathrm{fm})\end{array}$ & $\frac{\sigma}{T_{c}^{3}}$ \\
\hline 150 & 242.28 & 2.57 & 8.785 & 0.10 \\
170 & 263.24 & 2.60 & 9.282 & 0.10 \\
190 & 510.02 & 2.61 & 17.861 & 0.10 \\
210 & 678.23 & 2.59 & 24.082 & 0.10 \\
230 & 861.58 & 2.55 & 31.683 & 0.10 \\
250 & 1053.00 & 2.49 & 40.709 & 0.10 \\
270 & 1244.00 & 2.41 & 51.002 & 0.10 \\
290 & 1430.00 & 2.32 & 63.265 & 0.10 \\
\hline
\end{tabular}

\section{Result and Conclusion}

The surface tension at a suitable flow parameter such as $\beta_{q}=1 / 8, \beta_{g}=8 \beta_{q}$ is evaluated through the expression (2). Now surface tension $\sigma\left(M e V / \mathrm{fm}^{2}\right)$ is found to be increasing function with respect to the corresponding temperature of different droplet size in the both parameters. The increment is also observed with high gluon flow parameter at which the stability of QGP droplet is obtained near the hadronic phase. Moreover if we consider the ratio of surface tension to the cube of critical temperature, the result is found to be independent of the critical temperature and constancy is also observed in both cases of flow parameter. Thus surface tension $\sigma$ is found to be $0.10 T^{3}$ with the correction value in the potential. It increases from 
the earlier value $0.078 T^{3}$ to $0.10 T^{3}$. There is an improvement in the result and it becomes closer to lattice value $0.24 T^{3}$. This means that result with the correction factor in the mean field potential increases the value of surface tension to a very close result of lattice QCD (Iwasaki et al., 1994). It implies that the loop correction in the potential provides the stability of QGP dynamics with the appropriate flow parameters as well as the surface tension of the droplets with a good result.

\section{Acknowledgments}

The authors thank R. Ramanathan for his critical readings, discussions and suggestions in preparing the manuscript. We thank University Research Grant for financial support. One of the authors, Agam K Jha, also expresses gratitude to University of Delhi for financial support under Innovation Project.

\section{References}

1. Balian R and Block C (1970) Distribution of eigenfrequencies for the wave equation in a finite domain: I. Three-dimensional problem with smooth boundary surface Ann Phys (NY) 64401

2. Billoire A (1980) How Heavy Must Be Quarks in Order to Build Coulombic q anti-q Bound States Phys Lett B92 343

3. Brambilla N, Pineda A, Soto J and Vairo A (2001) The QCD potential at O(1/m) Phys Rev D63 014023

4. Christiansen MB and Madsen $J$ (1997) Inhomogeneity scale from the cosmological quark-hadron transition $J$ Phys G23 2039

5. Csernai LP, Kapusta JI and Osnes E (2003) Domain wall dynamics of phase interfaces Phys Rev D67 045003

6. Fischler W (1977) Quark - anti-Quark Potential in QCD Nucl Phys B129 157

7. Iwasaki Y, Kanaya K, Rummukainen K and Yoshie T (1994) Interface tension in quenched QCD Phys Rev D49 3540

8. Kapusta JI, Vischler AP and Venugopalan R (1995) Nucleation of quark - gluon plasma from hadronic matter Phys Rev C51 901

9. Linde AD (1983) Decay of the False Vacuum at Finite Temperature Nucl Phys B216 421

10. Mardor I and Svetitsky B (1991) Bubble free energy at the quark - hadron phase transition Phys Rev D44 878

11. Mustafa MG, Srivastava DK and Sinha B (1998) Effect of color singletness of quark gluon plasma in quark hadron phase transition Euro Phys J C5 711

12. Neergaard G and Madsen J (1999) Free energy of bubbles in the quark hadron phase transition Phys Rev D60 054011

13. Peshier A, Kämpfer B, Pavlenko OP and Soff G (1994) An Effective model of the quark - gluon plasma with thermal parton masses Phys Lett $\mathbf{B 3 3 7} 235$

14. Ramanathan R, Gupta KK, Jha AK and Singh SS (2007) The interfacial surface tension of a quark-gluon plasma fireball in a hadronic medium Pram J Phys 68757 
15. Ramanathan R, Mathur Y, Gupta KK and Jha AK (2004) A Simple statistical model for analysis of QGP-droplet (fireball) formation Phys Rev C70 027903

16. Satz H (1978) From Hadron To Quark Matter CERN-TH-2590 18pp

17. Shukla P and Mohanty AK (2001) Nucleation versus spinodal decomposition in a first order quark hadron phase transition Phys Rev C64 (2001) 054910

18. Shuryak EV (1973) Quantum Chromodynamics and the Theory of Superdense Matter Phys Rep 6171

19. Singh SS and Ramanathan R (2013) arXiv: 1308.3757v1

20. Smirnov AV, Smirnov VA and Steinhauser M (2008) Fermionic contributions to the three-loop static potential Phys Lett B668 293. 